\title{
A glycolysis-related two-gene risk model that can effectively predict the prognosis of patients with rectal cancer
}

\author{
Zhenzhen Liu' ${ }^{1 \dagger}$, Zhentao Liu² ${ }^{2 \dagger}$, Xin Zhou', Yongqu Lu', Yanhong Yao², Wendong Wang ${ }^{1}$, Siyi Lu', \\ Bingyan Wang ${ }^{1}$, Fei $\mathrm{Li}^{1}$ and Wei Fu${ }^{1 *}$
}

\begin{abstract}
Background: Aerobic glycolysis is an emerging hallmark of cancer. Although some studies have constructed glycolysis-related prognostic models of colon adenocarcinoma (COAD) based on The Cancer Genome Atlas (TCGA) database, whether the COAD glycolysis-related prognostic model is appropriate for distinguishing the prognosis of rectal adenocarcinoma (READ) patients remains unknown. Exploring critical and specific glycolytic genes related to READ prognosis may help us discover new potential therapeutic targets for READ patients.
\end{abstract}

Results: Three gene sets, HALLMARK_GLYCOLYSIS, REACTOME_GLYCOLYSIS and REACTOME_REGULATION_OF_GLYCOLYSIS_BY_FRUCTOSE_2_6_BISPHOSPHATE_METABOLISM, were both significantly enriched in both COAD and READ through glycolysis-related gene set enrichment analysis (GSEA). We found that six genes (ANKZF1, STC2, SUCLG2P2, P4HA1, GPC1 and PCK1) were independent prognostic genes in COAD, while TSTA3 and PKP2 were independent prognostic genes in READ. Glycolysis-related prognostic model of COAD and READ was, respectively, constructed and assessed in COAD and READ. We found that the glycolysis-related prognostic model of COAD was not appropriate for READ, while glycolysis-related prognostic model of READ was more appropriate for READ than for COAD. PCA and t-SNE analysis confirmed that READ patients in two groups (high and low risk score groups) were distributed in discrete directions based on the glycolysis-related prognostic model of READ. We found that this model was an independent prognostic indicator through multivariate Cox analysis, and it still showed robust effectiveness in different age, gender, M stage, and TNM stage. A nomogram combining the risk model of READ with clinicopathological characteristics was established to provide oncologists with a practical tool to evaluate the rectal cancer outcomes. GO enrichment and KEGG analyses confirmed that differentially expressed genes (DEGs) were enriched in several glycolysis-related molecular functions or pathways based on glycolysis-related prognostic model of READ.

Conclusions: We found that a glycolysis-related prognostic model of COAD was not appropriate for READ, and we established a novel glycolysis-related two-gene risk model to effectively predict the prognosis of rectal cancer patients.

Keywords: Glycolysis, Rectal cancer, TSTA3, PKP2, Prognosis

*Correspondence: fuwei@bjmu.edu.cn

†Zhenzhen Liu and Zhentao Liu have contributed equally to this work and share first authorship

${ }^{1}$ Department of General Surgery, Peking University Third Hospital, 49 Huayuan North Road, Haidian District, Beijing, People's Republic of China

Full list of author information is available at the end of the article

\section{Introduction}

Colorectal cancer (CRC), including colon cancer and rectal cancer, is the third most commonly diagnosed malignancy and ranks second in terms of mortality among cancers globally $[1,2]$. It is estimated that the incidence of CRC will increase to 2.5 million new cases worldwide in original author(s) and the source, provide a link to the Creative Commons licence, and indicate if changes were made. The images or other third party material in this article are included in the article's Creative Commons licence, unless indicated otherwise in a credit line to the material. If material is not included in the article's Creative Commons licence and your intended use is not permitted by statutory regulation or exceeds the permitted use, you will need to obtain permission directly from the copyright holder. To view a copy of this licence, visit http://creativecommons.org/licenses/by/4.0/. The Creative Commons Public Domain Dedication waiver (http://creativeco mmons.org/publicdomain/zero/1.0/) applies to the data made available in this article, unless otherwise stated in a credit line to the data. 
2035 due to the constant increase in developing countries and increased morbidity in younger people [3]. Although colon cancer and rectal cancer have been regarded as the same disease over the past decades, accumulating evidence has revealed that rectal cancer is different from colon cancer in many aspects, such as embryological origins, anatomy, risk factors, sensitivities to carcinogens, microbiota, and genetic subtypes [3-5]. Moreover, rectal cancer is the most common subtype among Asians, and the past decade has witnessed an increasing trend of early-onset rectal cancer (diagnosis before 50 years of age) $[2,4,6,7]$, which has constituted a formidable challenge in China and needs further investigation [4, 8]. Various biomarkers associated with the survival and prognosis of rectal cancer have been explored in the past. However, a single gene or biomarker cannot accurately predict the outcomes of cancer patients. The past decade has witnessed the soaring development of high-throughput sequencing [9-12], which has expanded our insight into the genetic alterations of rectal cancer and made it possible to establish multiple-gene predictive models using clinical and genetic data obtained from the public databases [13-15]. An mRNA-based gene signature for predicting rectal cancer patient outcomes is still needed in the present context.

Reprogramming energy metabolism is an emerging hallmark of cancer [16]. Even in the presence of oxygen, tumor cells give priority to glycolysis rather than mitochondrial oxidative phosphorylation for glucose catabolism, resulting in a state termed "aerobic glycolysis" or the "Warburg effect" [17]. A well-acknowledged rationale for the glycolytic switch in tumor cells is that increased glycolysis is employed by proliferating cells as a versatile product line, which continuously generates glycolytic intermediates for various biosynthetic pathways to satisfy the requirement for active cell proliferation [18]. Thus, exploring critical glycolytic genes related to rectal cancer prognosis may help us discover new potential therapeutic targets.

In this study, we found that a glycolysis-related prognostic model of colon cancer could not distinguish the prognosis of rectal cancer patients through bioinformatic analysis based on The Cancer Genome Atlas (TCGA) database. Moreover, we discovered the critical and specific glycolysis-related genes that participate in the development of rectal cancer using bioinformatic methods and established a practical model to predict rectal cancer patients' prognosis.

\section{Materials and methods Study design}

Our study compared glycolysis-related gene sets through gene set enrichment analysis (GSEA) and compared glycolysis-related independent prognosis genes between colon adenocarcinoma (COAD) and rectal adenocarcinoma (READ). Glycolysis-related prognostic model of COAD and READ was, respectively, constructed and assessed in COAD and READ. Assessment of the glycolysis-related READ prognostic model in READ patients was further performed through time-dependent receiver operating characteristic curve (time ROC) analysis, univariate and multivariate Cox analyses, principal component analysis (PCA) analysis, t-distributed stochastic neighbor embedding (t-SNE) analysis, Gene Ontology (GO) enrichment and Kyoto Encyclopedia of Genes and Genomes (KEGG) analyses. A nomogram combining the risk model of READ with clinicopathological characteristics was established for READ. The workflow for this study is shown in Additional file 1: Fig. S1.

\section{Data collection}

The mRNA expression profile of 144 READ and 8 adjacent normal rectal tissues were downloaded from the TCGA Genomic Data Commons (GDC) database (https://portal.gdc.cancer.gov/). The mRNA expression profiles of $398 \mathrm{COAD}$ and 39 adjacent normal colon tissues were also downloaded from the TCGA GDC database. The mRNA profiles were standardized by $\log 2$ transformation for further analysis. However, only 142 rectal cancer samples were documented with mRNA expression profiles and detailed clinicopathological information, including age, gender, American Joint Committee on Cancer (AJCC) TNM stage, T stage, $\mathrm{N}$ stage, $\mathrm{M}$ stage and survival status. The detailed clinical features of 142 rectal cancer patients are shown in Table 1. Patients whose follow-up time was absent, zero-days or unknown were excluded from the survival analysis. Finally, 135 READ patients and 363 COAD patients were enrolled for further survival analysis of glycolysis-related genes.

\section{Gene set enrichment analysis (GSEA)}

Gene sets enrichment analysis (GSEA) was conducted by GSEA software 4.10 from the Broad Institute [19] to identify the gene sets enriched between cancer tissues and adjacent normal tissues. A total of 12 glycolysis-related gene sets were downloaded from the Molecular Signatures Database (MSigDB) (https://www. gsea-msigdb.org/gsea/msigdb/genesets.jsp). To acquire a normalized enrichment score (NES), we conducted 1000 times gene set permutations for each analysis. When $|\mathrm{NES}|>1$, nominal $p$ value $<0.1$ and false discovery rate (FDR) $q$ value $<0.1$, the investigated gene sets were considered statistically significant. Glycolysis-related genes were extracted from the analysis performed by GSEA. We utilized the "limma" $\mathrm{R}$ package for analysis, which in turn yielded glycolysis-related differentially expressed 
Table 1 Clinical features in READ patients

\begin{tabular}{|c|c|}
\hline Variables & Patients, $n(\%)$ \\
\hline \multicolumn{2}{|l|}{ Sex } \\
\hline Male & 77 (54.2\%) \\
\hline Female & 65 (45.8\%) \\
\hline \multicolumn{2}{|l|}{ Age, years } \\
\hline$\leq 65$ & $73(51.4 \%)$ \\
\hline$>65$ & 69 (48.6\%) \\
\hline \multicolumn{2}{|l|}{ TNM stage } \\
\hline । & $26(18.3 \%)$ \\
\hline$\|$ & 41 (28.9\%) \\
\hline III & $45(31.7 \%)$ \\
\hline IV & $22(15.5 \%)$ \\
\hline Unknown & $8(5.6 \%)$ \\
\hline \multicolumn{2}{|l|}{ Tstage } \\
\hline $\mathrm{T} 1$ & $7(4.9 \%)$ \\
\hline $\mathrm{T} 2$ & 25 (17.6\%) \\
\hline T3 & 98 (69.0\%) \\
\hline T4 & $11(7.8 \%)$ \\
\hline Unknown & $1(0.7 \%)$ \\
\hline \multicolumn{2}{|l|}{ N stage } \\
\hline NO & $70(49.2 \%)$ \\
\hline N1 & $40(28.2 \%)$ \\
\hline N2 & $29(20.4 \%)$ \\
\hline N3 & $0(0.0 \%)$ \\
\hline NX & $2(1.4 \%)$ \\
\hline Unknown & $1(0.7 \%)$ \\
\hline \multicolumn{2}{|l|}{ M stage } \\
\hline MO & $106(74.6 \%)$ \\
\hline M1 & $21(14.9 \%)$ \\
\hline$M X$ & $13(9.1 \%)$ \\
\hline Unknown & $2(1.4 \%)$ \\
\hline \multicolumn{2}{|l|}{ Follow up time } \\
\hline$=0$ day or unknown & $7(4.9 \%)$ \\
\hline$>0$ day & $135(95.1 \%)$ \\
\hline
\end{tabular}

genes (DEGs), with filtering criteria of $p$ value $<0.05$ and $\log 2$ fold change $(\log 2 \mathrm{FC}) \neq 0$.

\section{Standardization of data processing and construction of the risk score model}

Univariate Cox regression analysis was first performed to screen out genes that were associated with overall survival $(\mathrm{OS})$ ( $p$ value $<0.05$ ). Subsequently, the identified genes were subjected to multivariate Cox regression analysis, and the corresponding regression coefficients related to OS were also acquired. When the values of hazard ratio (HR) are larger than 1, the corresponding factor is considered a risk factor. We used the "Cox. Zph" function to test the Cox proportional risk model in the "survival"
$\mathrm{R}$ package. When the $\mathrm{p}$ value of each covariate and global test is $>0.05$, it is considered that the Cox model conforms to the proportional risk hypothesis. In this study, glycolysis-related gene risk conformed to the proportional risk hypothesis and was constructed using the "coxph" function in the "survival" $\mathrm{R}$ package. The risk score formula is as follows: Risk score $=\sum_{i=1}^{n}$ expression of $G i \times \beta i$ (" $G$ " means gene; " $i$ " means order of genes; " $n$ " means the number of prognostic genes; " $\beta$ " means the regression coefficient of the corresponding gene after multivariate Cox analysis). According to the median risk score, all the included patients were divided into either the high-risk group and low-risk group.

\section{Assessment of the risk score model in rectal cancer patients}

Kaplan-Meier survival curves were drawn to show the survival rate difference between the two groups with a $p$ value $<0.05$ considered statistically significant. A ROC curve was plotted to evaluate the sensitivity and specificity of the risk score model. When the area under the curve (AUC) is $0.5-0.7$, the risk score model has acceptable efficiency. When the AUC is larger than 0.7, the risk score model has good accuracy. The "ggplot2" and "pheatmap" $\mathrm{R}$ packages were used to plot heat maps and survival status charts. PCA and t-SNE were performed to explore the distribution of different groups using the "Rtsne" R package. The "rms" R package was used to plot the nomogram for predicting the survival of rectal cancer patients. The time-dependent ROC curves and calibration plots were depicted to evaluate the efficiency of the nomogram.

\section{Functional enrichment analysis}

The "clusterProfiler" $\mathrm{R}$ package was utilized to conduct Gene Ontology (GO) and Kyoto Encyclopedia of Genes and Genomes (KEGG) analyses based on the DEGs $(|\log 2 \mathrm{FC}|>0.6, \mathrm{FDR}<0.05)$ between the high-risk and low-risk groups. $P$ values were adjusted with the Benjamini-Hochberg (BH) method.

\section{Statistical analysis}

All relevant statistical analyses were performed with $\mathrm{R}$ software (R4.0.2, https://www.r-project.org/).

\section{Result}

GSEA of glycolysis-related genes in COAD and READ

GSEA was, respectively, conducted to explore whether glycolysis-related gene sets were significantly enriched in the colon cancer and rectal cancer. Twelve glycolysis-related gene sets were investigated. Five gene sets, highlighted in boldface in Table 2, HALLMARK_GLYCOLYSIS, REACTOME_GLYCOLYSIS, 


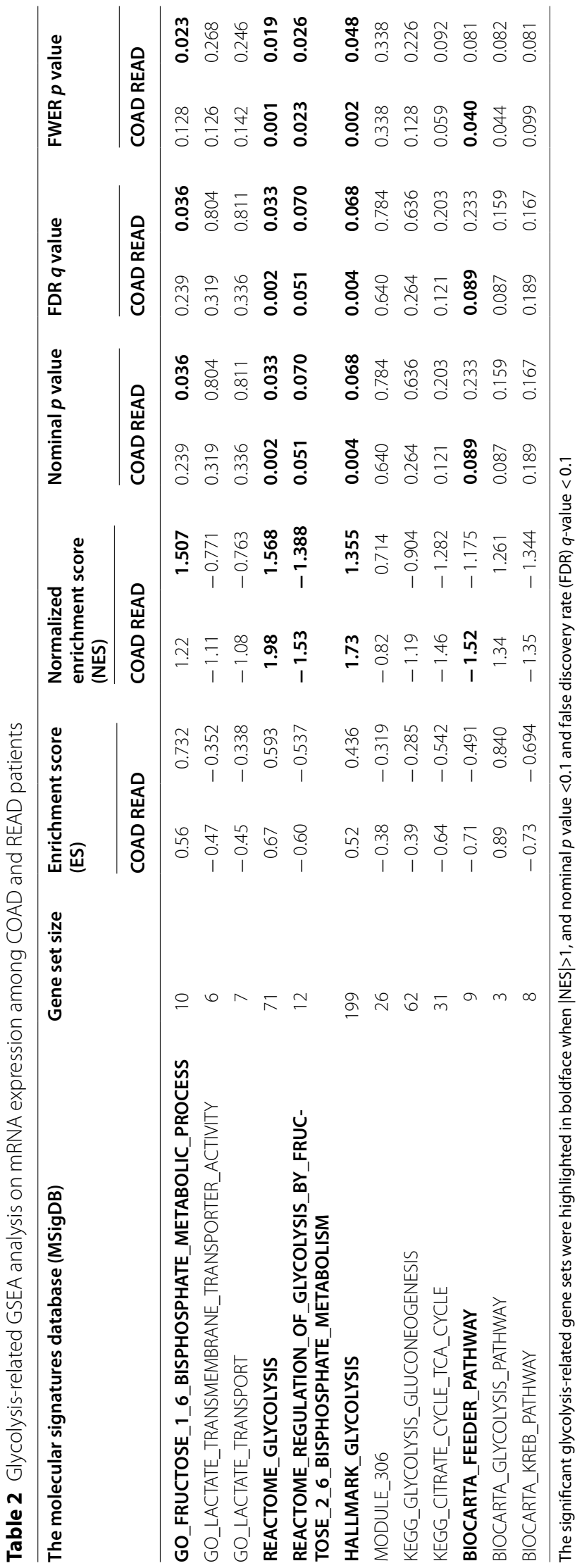


REACTOME_REGULATION_OF_GLYCOLYSIS_BY FRUCTOSE_2_6_BISPHOSPHATE_METABOLISM, BIOCARTA_FEEDER_PATHWAY and BIOCARTA GLYCOLYSIS_PATHWAY, were significantly enriched in the colon cancer samples $(|\mathrm{NES}|>1$, nominal $p$ value $<0.1$ and FDR $q$ value $<0.1$ ), as shown in Table 2 . Four gene sets, highlighted in boldface in Table 2, HALLMARK_GLYCOLYSIS, REACTOME_GLYCOLYSIS, GO_FRUCTOSE_1_6_BISPHOSPHATE_METABOLIC PROCESS, and REACTOME_REGULATION_OF_GLYCOLYSIS_BY_FRUCTOSE_2_6_BISPHOSPHATE_ METABOLISM, were significantly enriched in the rectal cancer samples ( $|\mathrm{NES}|>1$, nominal $p$ value $<0.1$ and FDR $q$ value $<0.1)$, as shown in Table 2 . Three gene sets, HALLMARK_GLYCOLYSIS, REACTOME_GLYCOLYSIS, and

Table 3 Glycolysis-related prognostic genes in COAD and READ by univariate analysis

\begin{tabular}{lllll}
\hline Cancer type & Gene & HR & 95\% Cls & P value \\
\hline COAD & STC1 & 1.396 & $1.053-1.849$ & 0.020 \\
& ANKZF1 & 1.963 & $1.110-3.470$ & 0.020 \\
& STC2 & 1.251 & $1.010-1.548$ & 0.040 \\
& SDHB & 0.490 & $0.301-0.799$ & 0.004 \\
& SUCLG2P2 & 0.109 & $0.034-0.345$ & 0.000 \\
& P4HA1 & 1.482 & $1.062-2.067$ & 0.021 \\
& PPFIA4 & 5.078 & $2.154-11.970$ & 0.000 \\
READ & GPC1 & 1.406 & $1.075-1.839$ & 0.013 \\
& PCK1 & 0.802 & $0.655-0.981$ & 0.032 \\
& TSTA3 & 2.661 & $1.301-5.443$ & 0.007 \\
& IDH3A & 0.364 & $0.167-0.794$ & 0.011 \\
& PKP2 & 0.419 & $0.238-0.737$ & 0.002 \\
& ACO2 & 0.493 & $0.290-0.837$ & 0.008 \\
\hline
\end{tabular}

REACTOME_REGULATION_OF_GLYCOLYSIS_BY_ FRUCTOSE_2_6_BISPHOSPHATE_METABOLISM, were both significantly enriched in the colon cancer and rectal cancer.

\section{Comparison of glycolysis-related prognostic genes between COAD and READ}

A total of 316 genes in the above gene sets were included for further analysis. Subsequently, 199 glycolysis-related DEGs in READ and 254 glycolysis-related DEGs in COAD were, respectively, identified with $p$ value $<0.05$ and $|\log 2 \mathrm{FC}|>0$ as the cutoff standard. To further, respectively, explore prognostic genes in COAD and READ, glycolysis-related DEGS were analyzed by univariate Cox regression. Nine genes (STC1, ANKZF1, STC2, SDHB, SUCLG2P2, P4HA1, PPFIA4, GPC1 and $P C K 1)$ in COAD and 4 genes (TSTA3, IDH3A, PKP2 and $A C O 2)$ in READ were found to be significantly associated with overall survival (OS) ( $p$ value $<0.05$ ) (Table 3 ). No same genes were both significantly associated with OS in COAD and READ. Subsequently, multivariate Cox regression analysis was, respectively, performed in COAD and READ. Six genes including ANKZF1, STC2, SUCLG2P2, P4HA1, GPC1 and PCK1 were proven to be independent prognostic factors in COAD (Table 4). Two genes, TSTA 3 and PKP2, were proven to be independent prognostic factors in READ (Table 4). We compared the expression levels of the above genes between the cancer tissues and adjacent normal tissues, and found that $A N K Z F 1, S T C 2$ and P4HA1 were significantly upregulated, while SUCLG2P2 and PCK1 were downregulated in both COAD and READ among the independent prognostic genes of COAD (Fig. 1). Although GPC1 was significantly upregulated in COAD, it was not upregulated

Table 4 Glycolysis-related gene risk model for predicting READ prognosis

\begin{tabular}{|c|c|c|c|c|c|}
\hline Cancer type & Gene & $\beta$ (coef) & HR & $95 \% \mathrm{Cl}$ & $P$ value \\
\hline \multirow[t]{7}{*}{ COAD } & ANKZF1 & 0.543 & 1.722 & $0.972-3.049$ & 0.062 \\
\hline & STC2 & 0.257 & 1.293 & $1.030-1.624$ & 0.027 \\
\hline & SUCLG2P2 & -2.124 & 0.120 & $0.035-0.407$ & 0.001 \\
\hline & P4HA1 & 0.359 & 1.432 & $1.013-2.025$ & 0.042 \\
\hline & GPC1 & 0.326 & 1.385 & $1.040-1.843$ & 0.026 \\
\hline & PCK1 & -0.256 & 0.774 & $0.632-0.950$ & 0.014 \\
\hline & \multicolumn{5}{|c|}{$\begin{array}{l}\text { Risk model formula }=\sum_{i=1}^{n} \text { expression of } G i \times \beta i=\text { expression of } A N K Z F 1 \times 0.543+\text { expression of } \\
\text { STC2 } \times(0.257)+\text { expression of } S U C L G 2 P 2 \times(-2.124)+\text { expression of } P 4 H A 1 \times(0.359)+\text { expression of } \\
G P C 1 \times(0.326)+\text { expression of } P C K 1 \times(-0.256)\end{array}$} \\
\hline Cancer type & Gene & $\beta$ (coef) & HR & $95 \% \mathrm{Cl}$ & $P$ value \\
\hline \multirow[t]{3}{*}{ READ } & TSTA3 & 0.752 & 2.121 & $1.067-4.218$ & 0.032 \\
\hline & PKP2 & -0.725 & 0.484 & $0.274-0.856$ & 0.013 \\
\hline & \multicolumn{5}{|c|}{ Risk model formula $=\sum_{i=1}^{n}$ expression of $G i \times \beta i=$ expression of TSTA3 $\times 0.752+$ expression of PKP $2 \times(-0.725)$} \\
\hline
\end{tabular}




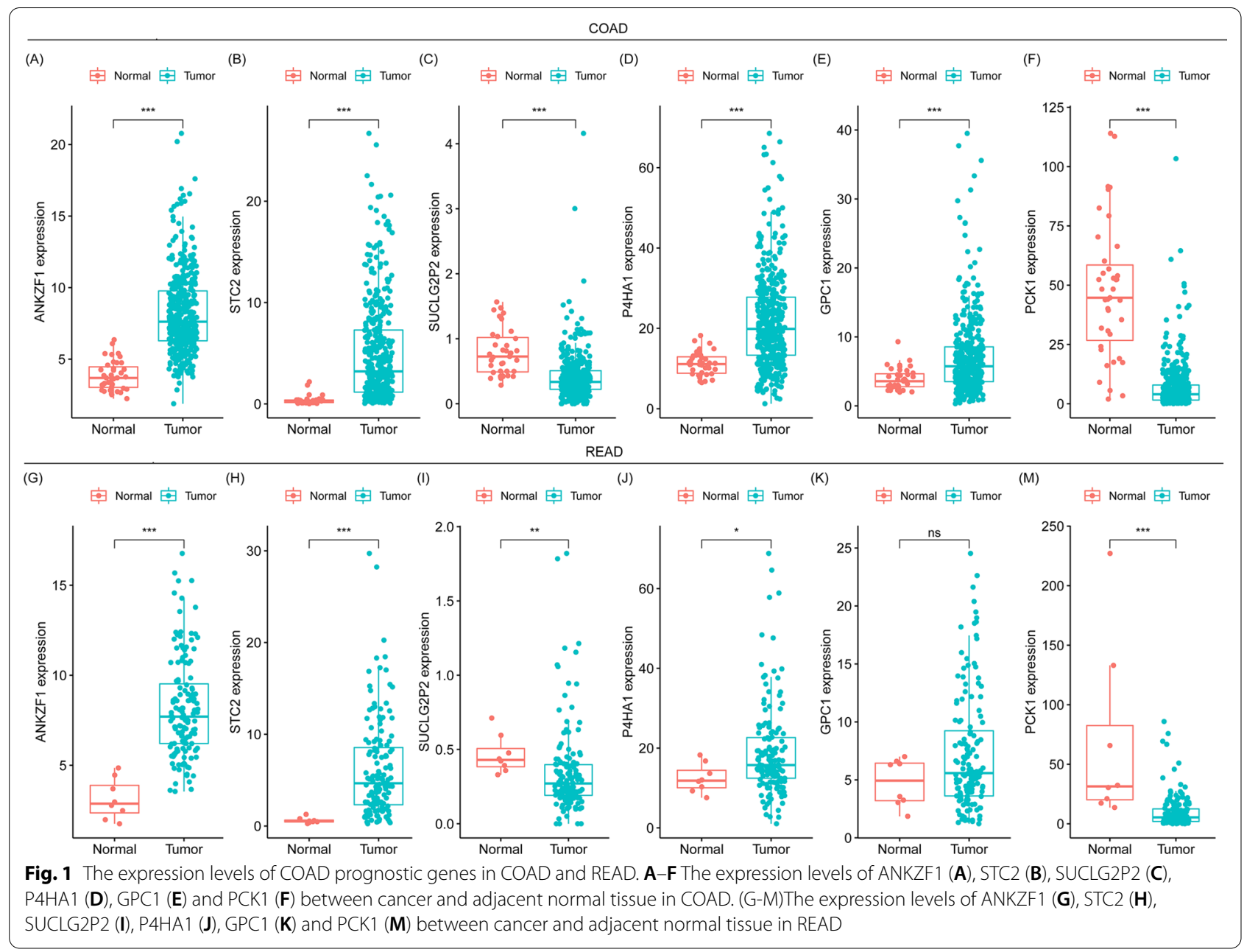

in READ (Fig. 1). Among the independent prognostic genes of READ, we found that TSTA3 was significantly upregulated while PKP2 was downregulated in both COAD and READ (Fig. 2). Taken together, these results indicated that there existed different glycolysis-related prognosis genes between COAD and READ.

\section{The glycolysis-related COAD prognostic model is not appropriate for READ}

Above all, we established a risk score model based on the independent prognostic genes of COAD to, respectively, evaluate COAD and READ patient survival. The risk score model formula is as follows: risk score $=$ expression of $A N K Z F 1 \times(0.543)+$ expression of $S T C 2 \times(0.257)$ + expression of $S U C L G 2 P 2 \times(-2.124)+$ expression of P4HA1 × $(0.359)+$ expression of GPC1 $\times(0.326)+$ expression of $P C K 1 \times(-0.256)$ (Table 4).
COAD patients were divided into a high-risk group and a low-risk group according to the median risk score of the whole COAD patients. We found that COAD patients with a high-risk score had a shorter OS than those with a low-risk score (Fig. 3A). A ROC curve was further drawn to evaluate the reliability of this prognostic model. The AUC of this predictive model accumulated steadily over time and came to 0.781 at 3 years, indicating a satisfactory sensitivity and specificity of this model in predicting survival for COAD patients (Fig. 3C). We wondered whether a risk score model of COAD could distinguish the prognosis of READ patients. The risk score of READ patients was calculated according to the risk model formula of COAD. READ patients were also divided into a high-risk group and a low-risk group according to the median risk score of the whole COAD patients. We found that the glycolysis-related prognostic model of COAD could not distinguish the prognosis of READ patients 


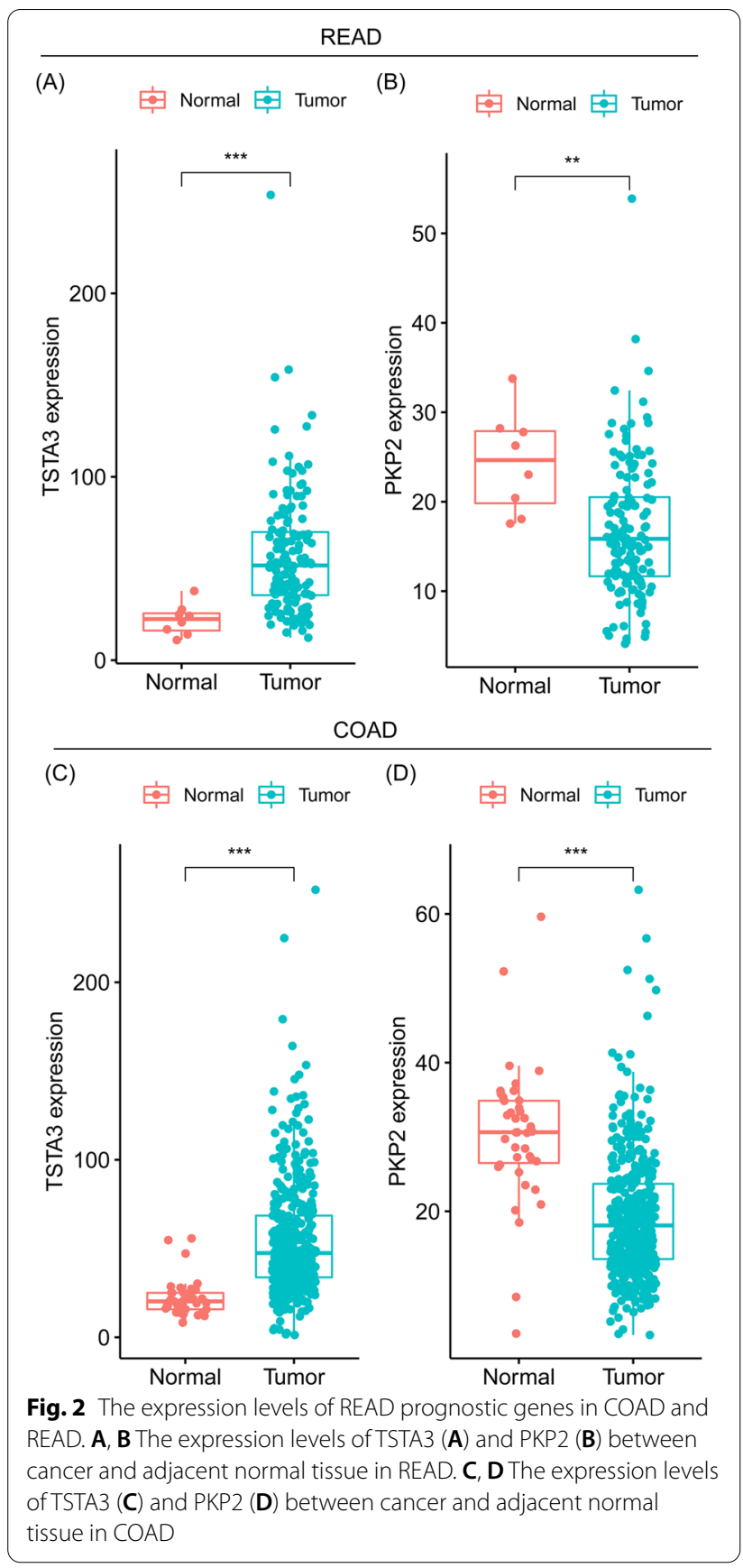

according to Kaplan-Meier survival analysis (Fig. 3C). Moreover, the AUC of the ROC curve was 0.554 at 3 years in READ (Fig. 3D), which was lower than the AUC in COAD. In addition, READ patients were divided into a high-risk group and a low-risk group according to the median risk score of the whole READ patients based on COAD prognostic model. The survival curves of the high-risk group and low-risk group crossed with the $p$ value $>0.05$ in READ (Additional file 1: Fig. S2A). These results indicated that the glycolysis-related prognostic model of COAD is not appropriate for READ.

\section{The glycolysis-related READ prognostic model is suitable for READ}

Subsequently, we established risk score model based on the independent prognostic gene of READ to, respectively, evaluate READ and COAD patients' survival. The risk score model formula is as follows: risk score $=$ expression of TSTA $3 \times(0.752)+$ expression of $P K P 2 \times(-0.725)($ Table 4$)$. READ patients were divided into a high-risk group and a low-risk group according to the median risk score of the whole READ patients. We found that READ patients with a high-risk score had a shorter OS than those with a low-risk score (Fig. 4A). A ROC curve was drawn to evaluate the reliability of this prognostic model. The AUC of this predictive model accumulated steadily over time and came to 0.783 at 3 years, indicating a satisfactory sensitivity and specificity for this model in predicting the survival of READ patients (Fig. 4C). We wondered whether the risk score model of READ could distinguish the prognosis of COAD patients. Risk score of COAD patients was calculated according to the risk model formula of READ. COAD patients were also divided into a high-risk group and a low-risk group according to the median risk score of the whole READ patients. We found that the survival curves of the high-risk group and low-risk group crossed despite the $p$ value $<0.05$ in COAD (Fig. 4B). In addition, the AUC was 0.542 at 3 years in COAD (Fig. 4D), which was lower than the AUC in READ. In addition, COAD patients were divided into a high-risk group and a low-risk group according to the median risk score of the whole COAD patients based on READ prognostic model. The survival curves of the high-risk group and low-risk group still crossed with the $p$ value $>0.05$ in COAD (Additional file 1: Fig. S2B). These results indicated that the READ risk score model was more appropriate for READ than for COAD.

To provide visualization of risk score, survival status, and gene expression in READ. The risk score, survival status, and gene expression of the two genes in each READ patient are shown in Fig. 5A-C. PCA and t-SNE analysis confirmed that READ patients in the two subgroups were distributed in discrete directions (Fig. 5D-E).

\section{Assessment of the glycolysis-related READ prognostic model in READ patients}

To evaluate this prediction model's clinical utility, univariate and multivariate Cox proportional hazards regression analyses were conducted to compare this two-gene risk model with common clinicopathological features. 


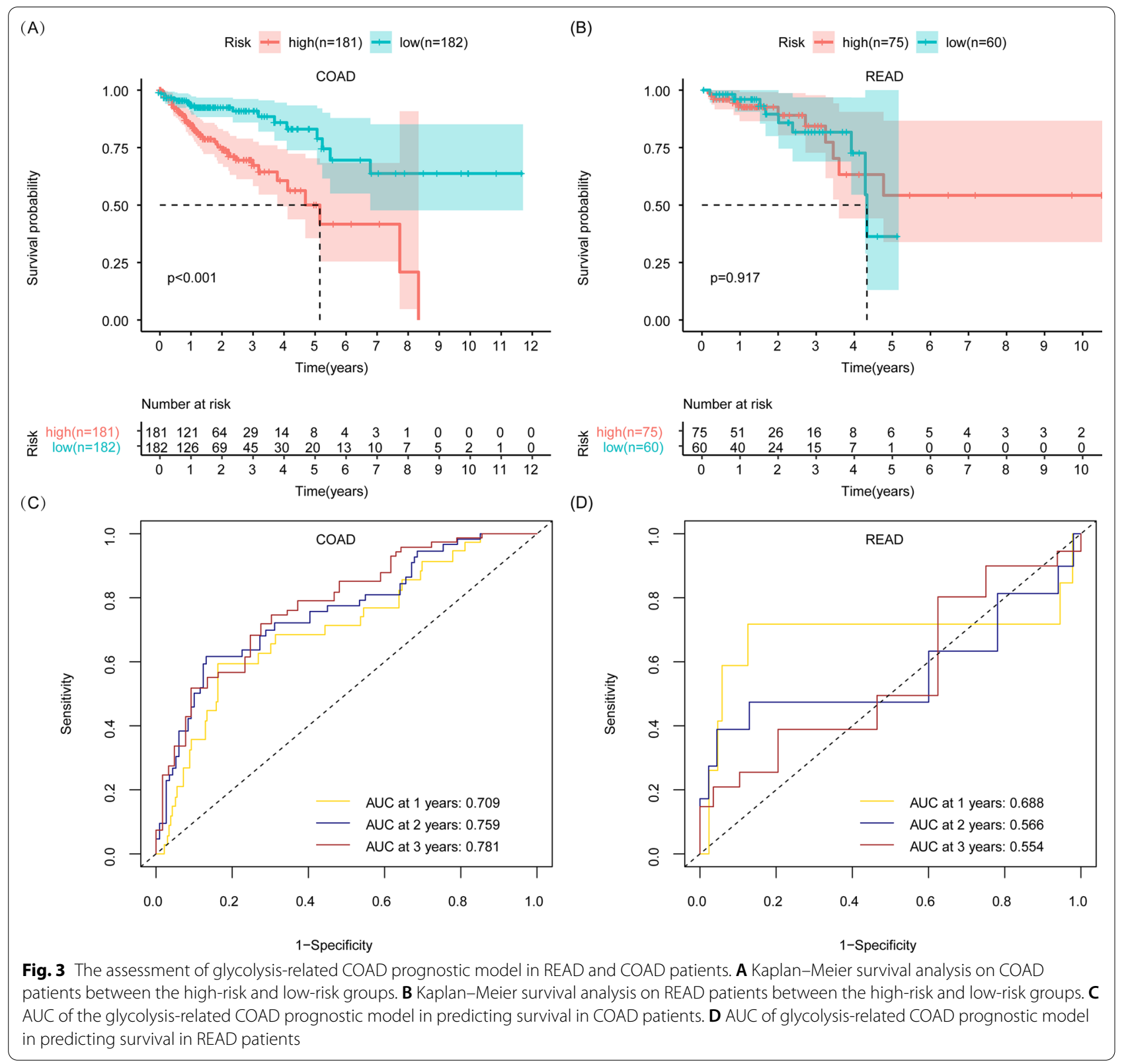

Univariate analysis showed that age, TNM stage, and risk score were associated with rectal cancer patient survival (Fig. 6A). These three factors were further proven to be independent prognostic indicators in the subsequent multivariate Cox analysis (Fig. 6B), indicating that the two-gene risk model can serve as a promising tool for predicting the prognosis of patients with rectal cancer. The Kaplan-Meier curves illustrated that patients with older age, higher $\mathrm{N}$ stage, metastasis status, higher TNM stage, and a higher risk score had a poor prognosis. In contrast, gender and $\mathrm{T}$ stage had no effect on prognosis of rectal cancer patients (Fig. $6 \mathrm{C}-\mathrm{H}$ ).
To further verify the effectiveness of this two-gene model in predicting prognosis in rectal cancers, the patients were classified into different subgroups according to age ( $\leq 65$ years vs $>65$ years), gender (female vs male), T stage (T1-2 vs T3-4), N stage (N0 vs N1-2), $\mathrm{M}$ stage (M0 vs M1) and AJCC TNM stage (stage I-II vs stage III-IV). They were subsequently divided into the high-risk score and low-risk score groups based on the median risk score. Interestingly, the two-gene risk model still showed robust effectiveness in the different age, gender, $\mathrm{M}$ stage, and AJCC stage subgroups (Fig. 7AF). However, high-risk scores did not suggest a poor 


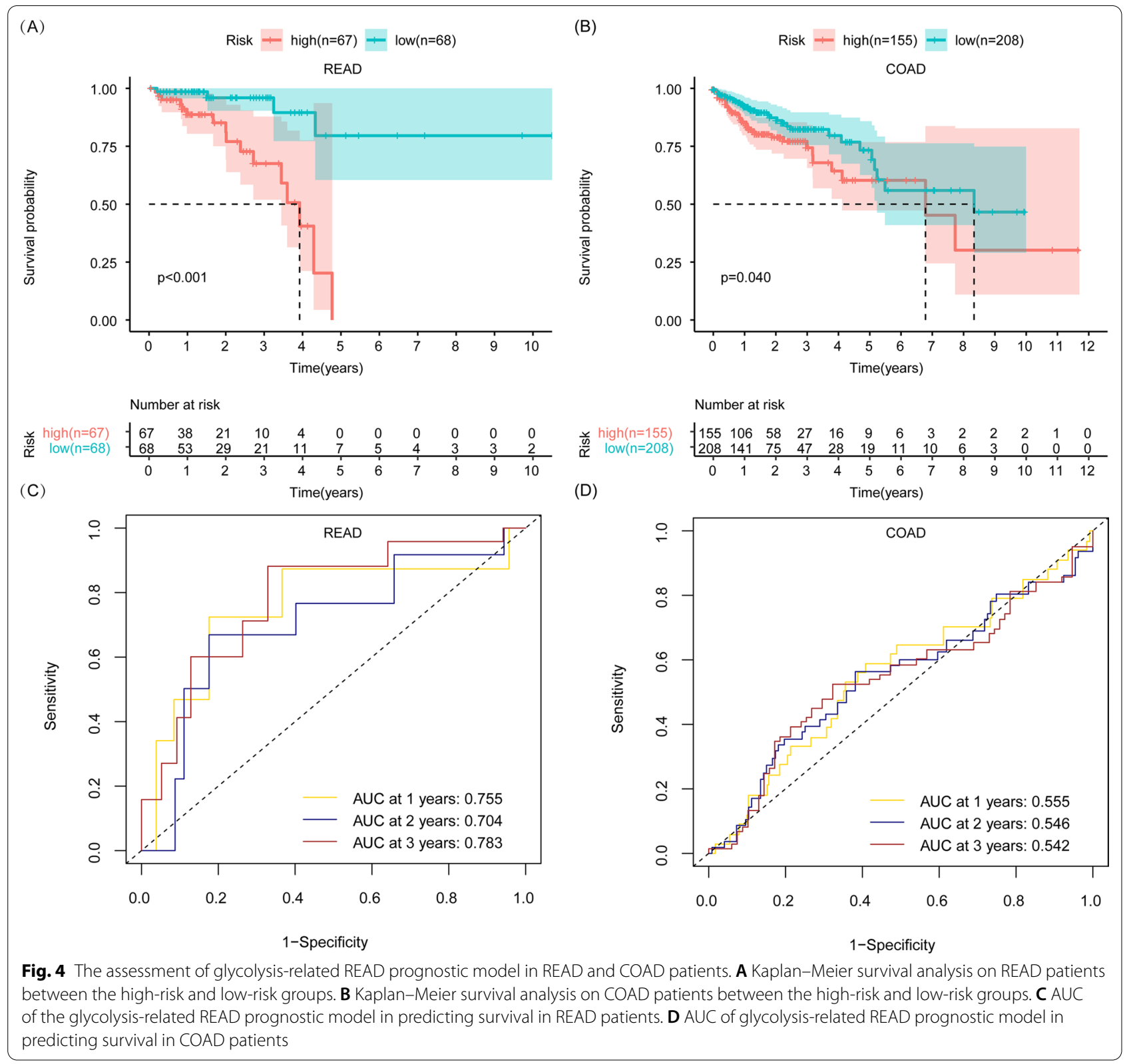

prognosis in the N0 stage and T1-2 stage subgroups (Fig. 7A-F), indicating that the risk score model may be a more effective prognostic marker in the late stages of rectal cancer.

\section{Construction and evaluation of a nomogram incorporating the two-gene risk score model and clinical features}

To provide a clinically available and practical tool for oncologists to estimate rectal cancer patients' survival time, we established a nomogram combining risk score with clinicopathological characteristics (age and TNM stage) (Fig. 8A). Calibration plots suggested that the nomogram fitted well compared with the ideal model representing by the $45^{\circ}$ line, as shown in Fig. $8 \mathrm{~B}-\mathrm{D}$. The C-index of the nomogram was 0.852 , which indicated that this nomogram had an outstanding stability. ROC curves showed that the AUCs of the nomogram at 1 , 2 , and 3 years was $0.838,0.849$, and 0.859 , respectively, which were better than those of the clinical factors or risk score model alone (Fig. 8E). 
(A)

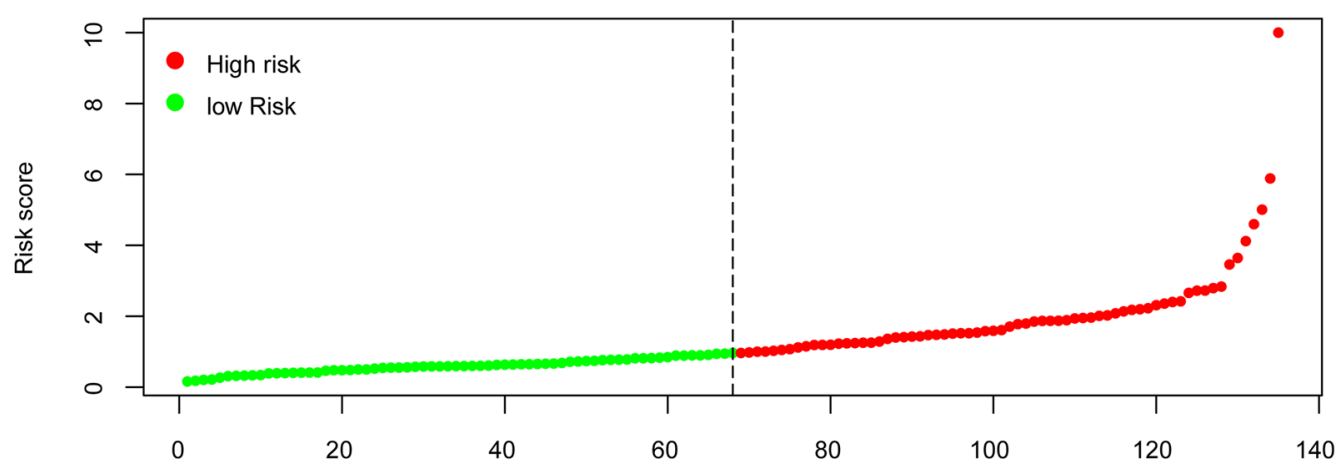

(B)

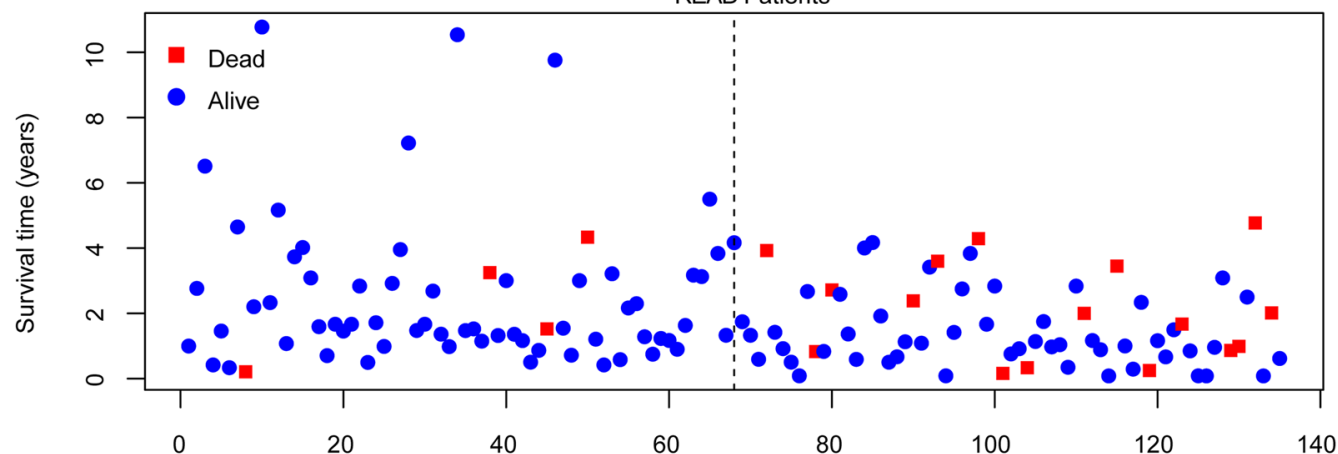

(C)

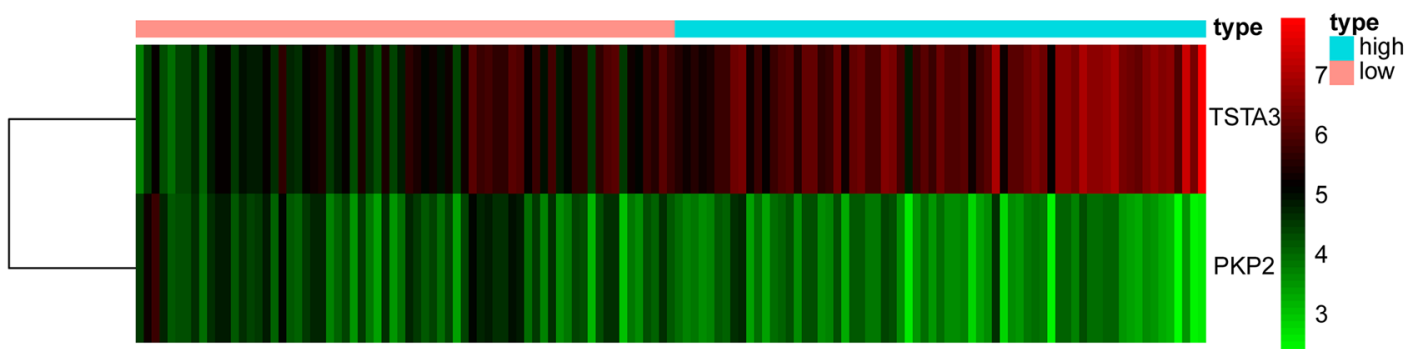

(D)

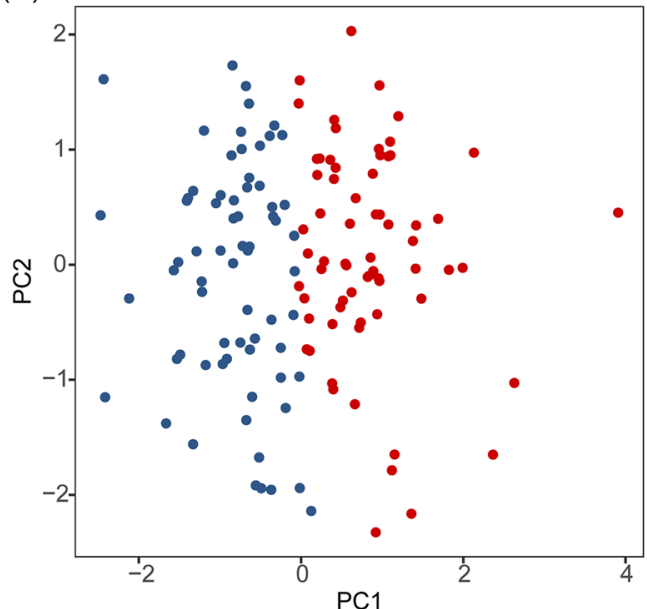

(E)

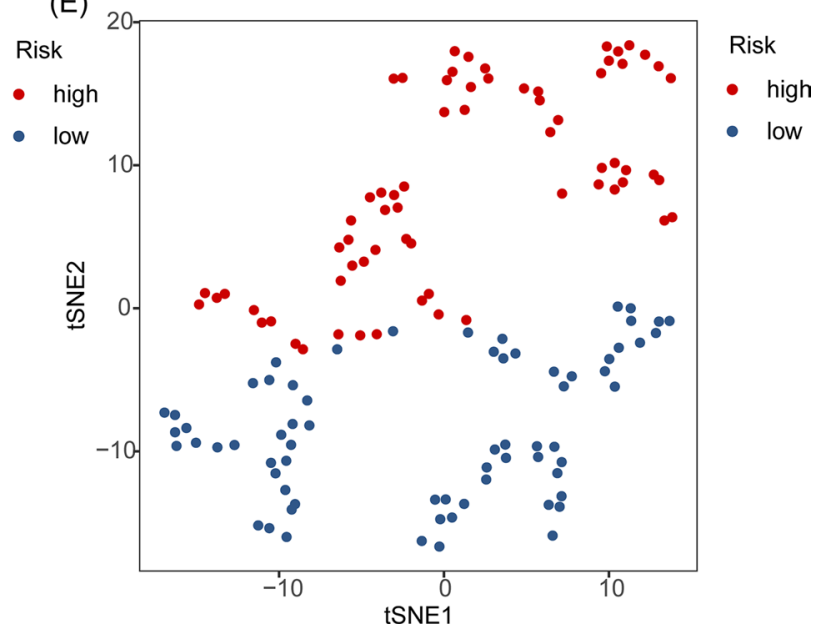

Fig. 5 The characteristic of glycolysis-related READ prognostic model. A Distribution of risk scores of each READ patient. B Correlation between survival time and survival status of each patient. C The expression pattern of TSTA3 and PKP2 between high-risk and low-risk groups. D PCA analysis of READ patient based on glycolysis-related READ prognostic model. E t-SNE analysis of READ patient based on glycolysis-related READ prognostic model. The dashed line represents the median risk score in READ patients 
Functional analyses between high-risk and low-risk groups in patients with rectal cancer

To elucidate the different biological functions or pathways that might play potential roles in the high-risk group and low-risk group, GO enrichment and KEGG analyses were performed using the DEGs $(|\log 2 \mathrm{FC}| \geq 0.6, \mathrm{FDR}<0.05)$ between the two groups. As expected, the DEGs were enriched in several glycolysis-related molecular functions or pathways, such as oxidative phosphorylation $(p$ value $<0.05$ ) (Fig. 9A-D). These results indicated that glycolysis-related molecular functions or pathways were indeed different between high-risk group and low-risk group according to glycolysis-related READ prognostic model.

\section{Discussion}

Cancer metabolism, especially glucose metabolism, has drawn great attraction in oncology research during the in recent decades [16-18]. Enhanced aerobic glycolysis, which serves as a metabolic driver, has been proven to promote tumor development and aggressiveness in CRC cells, and further results in resistance to treatment [20, 21]. In our research, we compared glycolysis-related gene sets between COAD and READ. Three gene sets, HALLMARK_GLYCOLYSIS, REACTOME_GLYCOLYSIS, and REACTOME_REGULATION_OF_GLYCOLYSIS_BY_ FRUCTOSE_2_6_BISPHOSPHATE_METABOLISM, were significantly enriched in both COAD and READ. We also compared glycolysis-related independent prognostic genes between COAD and READ. We found that six genes including ANKZF1, STC2, SUCLG2P2, P4HA1, GPC1 and $P C K 1$ were independent prognostic genes in COAD, while TSTA 3 and PKP2, were independent prognostic genes in READ. These results indicated that COAD might have different glycolysis-related prognostic gene signatures than READ. We, respectively, constructed glycolysis-related prognostic models in COAD and READ and, respectively, assessed the efficiency of each model in COAD and READ. We found that the glycolysis-related prognostic model of COAD was not appropriate for READ, while glycolysisrelated prognostic model of READ was more appropriate for READ than COAD. These results indicated that COAD might have many different patterns such as genomic signatures, drug efficacy, and prognosis compared with READ.

We also developed a two-gene signature (TSTA3 and $P K P 2)$ to predict the prognosis of rectal cancer, which was shown to be an independent prognostic indicator through multivariate Cox analysis. Subgroup analysis confirmed that the READ glycolysis-related prognostic model still showed robust effectiveness in the different age, gender, $M$ stage, and AJCC stage subgroups. Moreover, a nomogram integrating the risk model and clinicopathological factors was depicted to provide clinicians with a practical tool for predicting the prognosis of rectal cancer patients.

Recent research has revealed that traditional clinicopathological factors are inadequate for accurate cancer prognosis predictions [13, 14]. With the rapid development of high-throughput sequencing and the accumulation of cancer genomic data, molecular signatures based on data mining in the public databases have become a reality to predict the outcome of cancer patients, demonstrating higher sensitivity and specificity than the traditional single genes or markers models [11, 12, 15]. Glycolysis-related risk scores have shown excellent performance in predicting prognosis in various solid tumors [14, 22-27]. Liu et al. [23] developed a four-gene signature (AGRN, AKR1A1, DDIT4, and HMMR) related to glycolysis to predict the lung adenocarcinoma patient outcomes that showed desirable accuracy. Another fourgene glycolytic signature (NUP205, NUPL2, PFKFB1, and $P K M)$ showed excellent performance in predicting the OS of bladder cancer patients [27]. Similarly, Chen et al. established a risk score model containing seven glycolysis-related genes (PPARGC1A, DLAT, 6PC2, P4HA1, $S T C 2, A N K Z F 1$, and GPC1) in their recent study that can effectively predict the outcome of colon adenocarcinoma [14]. However, the genes identified in the COAD background in Chen's research are different from the genes identified in our present study, resulting in two different risk models, which further validate the mainstream view that colon cancer and rectal cancer are two diseases [3-5].

Plakophilin-2 (PKP2) was initially identified as a desmosomal protein, but further studies have revealed that it is localized in the cytoplasm and nucleus as well [28]. $P K P 2$ expression is upregulated in many cancers, such as lung [29], ovarian [30], glioma [31], and bladder cancers [32]. Increased PKP2 expression was also related to a malignant phenotype and poor prognosis in some cancers [29-32]. Arimoto et al. found that PKP2 enhanced the dimerization of EGFR and activated downstream signaling pathways, which further promoted cell proliferation and tumor metastasis [33]. Moreover, PKP2 may

(See figure on next page.)

Fig. 6 The cox analysis on prognostic model and survival analysis on different clinical features in READ. A Forest plot for the risk score model compared with other clinical features in READ patients by univariate analyses. B Forest plot for the risk score model compared with other clinical features in READ patients by multivariate analyses. $\mathbf{C}-\mathbf{H}$ Kaplan-Meier survival analysis for different clinical features, including age (C), gender (D), TNM stage $(\mathbf{E})$, T stage $(\mathbf{F})$, N stage $(\mathbf{G})$, and $\mathbf{M}$ stage $(\mathbf{H})$, in READ patients 


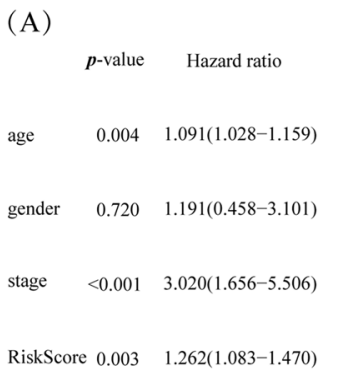

(C)

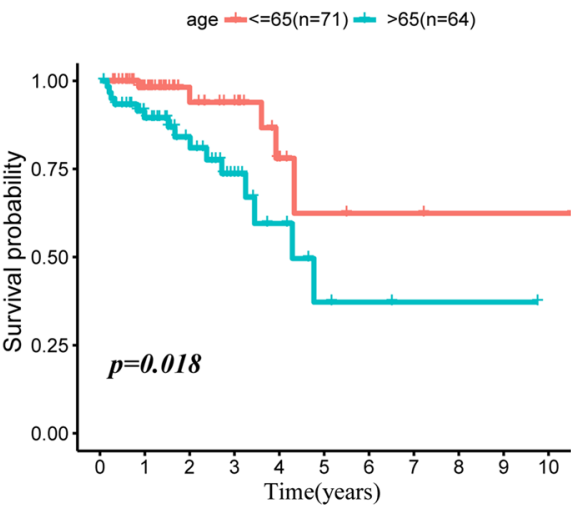

(E)

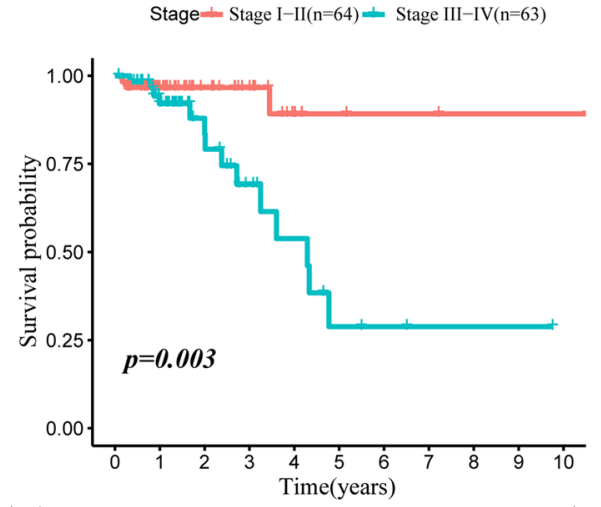

( $G)$

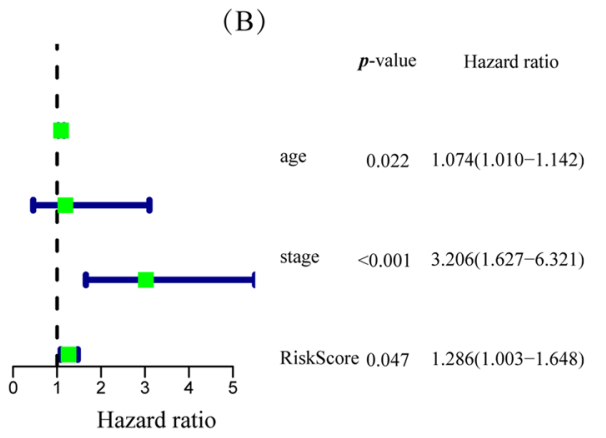

(D)

(F)

(H)

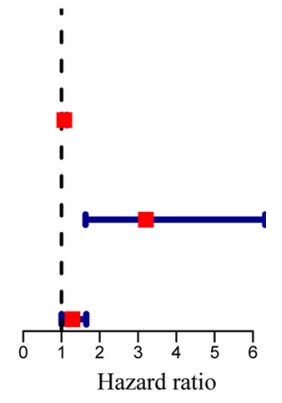

Hazard ratio
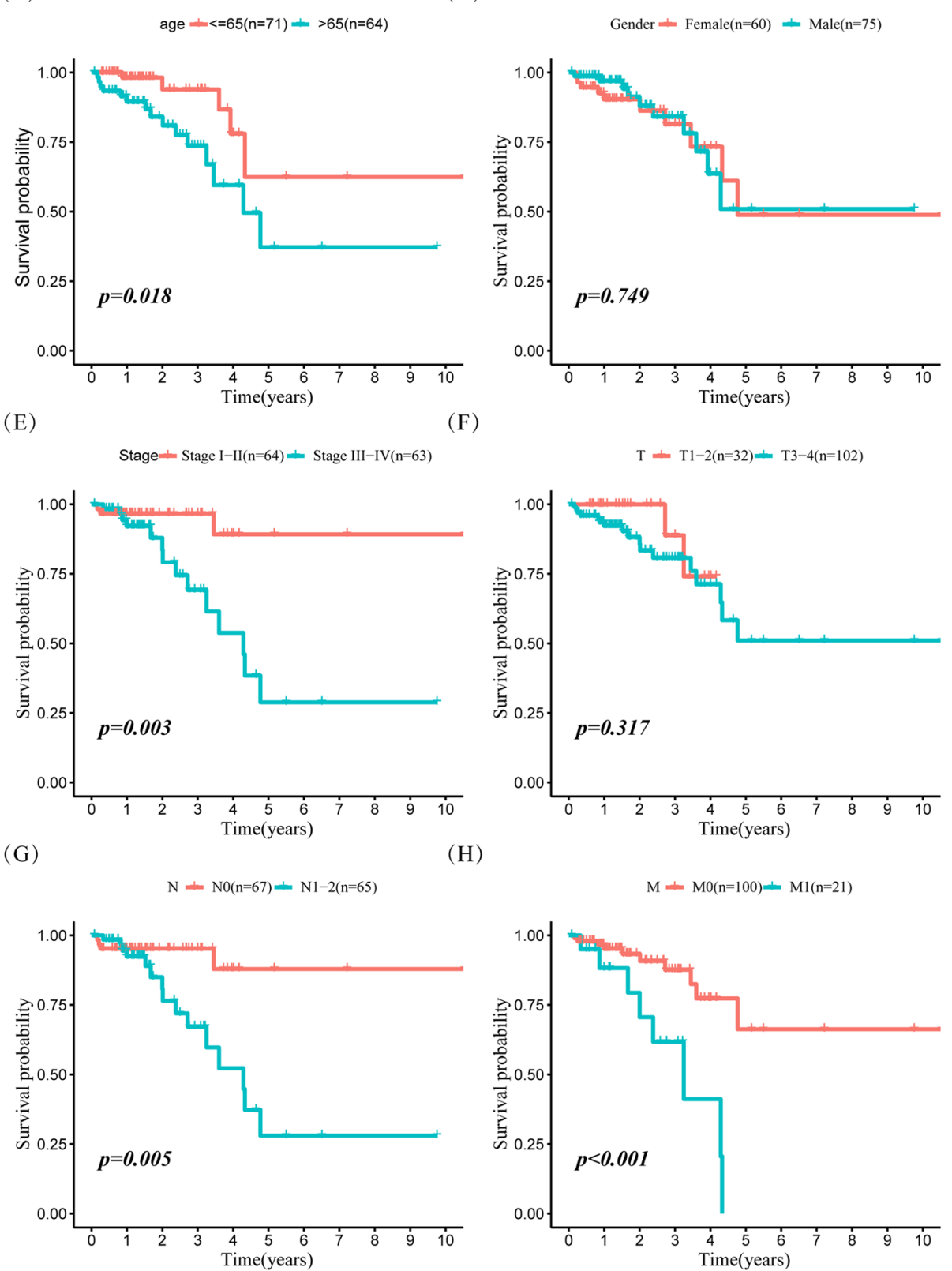

Fig. 6 (See legend on previous page.) 


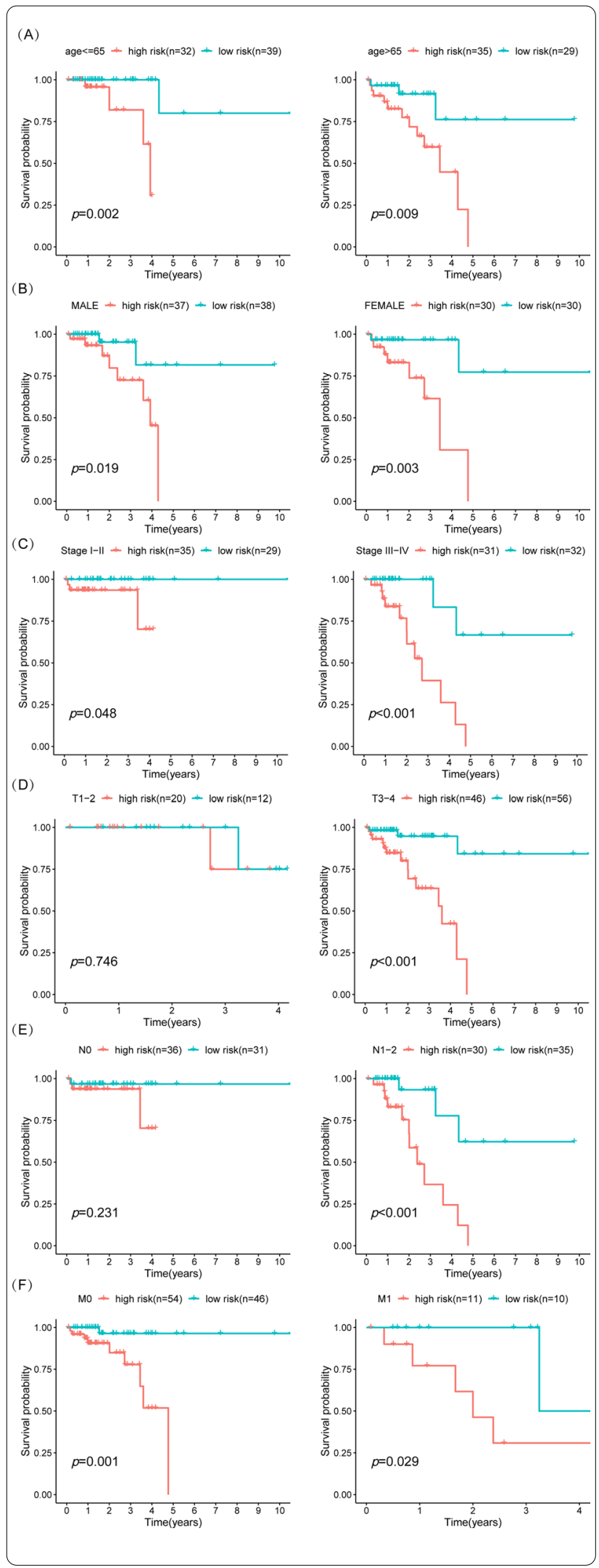

Fig. 7 Hierarchical survival analysis of glycolysis-related READ prognostic model in READ. Hierarchical survival analysis on clinical features [age (A), gender (B), TNM stage $(\mathbf{C})$, T stage (D), N stage $(\mathbf{E})$, and $M$ stage $(\mathbf{F})$.]

function as a feedback inhibitor of Wnt/ $\beta$-catenin signaling in CRC stromal fibroblasts and regulate Wnt activity in CRC cells [34]. Our present study found that PKP2 was downregulated in rectal cancer tissues compared with the adjacent normal tissues. Low PKP2 expression was correlated with a poor prognosis, indicating a distinct role of $P K P 2$ in rectal cancer development, which needs to be further investigated.

Tissue-specific transplantation antigen P35B (TSTA3), also called GDP-L-fucose synthase, is one of the two rate-limiting enzymes in the de novo synthesis pathway of GDP-L-fucose [35-37]. Serving as a one common glycosylation modification by conjugating fucose to protein- or lipid-bound oligosaccharides, fucosylation is dysregulated in various cancers and is related to carcinogenesis, invasion, and metastasis [38, 39]. The process of fucosylation may provide novel targets for cancer therapeutics [40]. Recently, Zhang et al. [41] found that increased TSTA3 expression could promote esophageal cancer progression by fucosylation of $L A M P 2$ and $E R B B 2$ and could predict a poor prognosis [42]. Wang et al. [43] found that knocking out TSTA3 in mice could lead to fucosylation deficiency and further result in colitis and adenocarcinoma. In contrast, we discovered that TSTA3 was upregulated in rectal cancer tissues compared to adjacent normal tissues. High expression was correlated with a poor prognosis, indicating that TSTA3 may function as a tumor suppressor in rectal cancer. Since TSTA3 plays different roles in diverse kinds of cancer [42-45], the accurate biological function of TSTA3 in rectal cancer remains to be elucidated.

The two-gene risk model associated with glycolysis demonstrated effective performance in predicting the clinical outcomes of patients with rectal cancer. However, we could not find a large sample database of highthroughput gene expression READ for validation and we found two more than 100 samples colorectal cancer (CRC) database with microarray gene expression database and OS information in Gene Expression Omnibus (GEO) database for validation. The high-risk group had a higher OS rate than that of low-risk group with the $p$ value $=0.029$ according to GSE39582 database (Additional file 1: Fig. S3A). The survival curves of the high-risk group and low-risk group crossed with the 
(A)

Points

age

stage

riskScore

Total Points

1-year survival

2-year survival

3-year survival

(B)

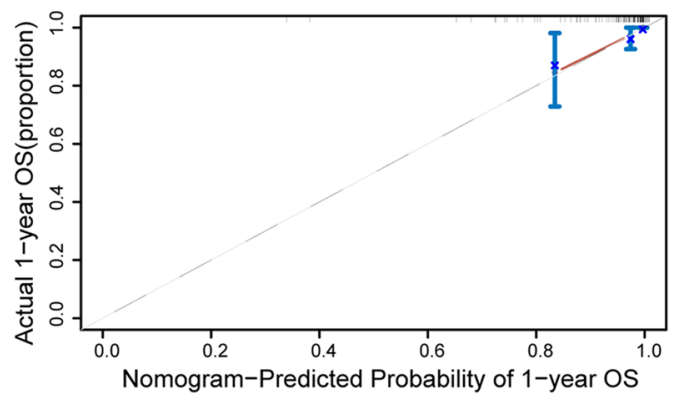

(D)

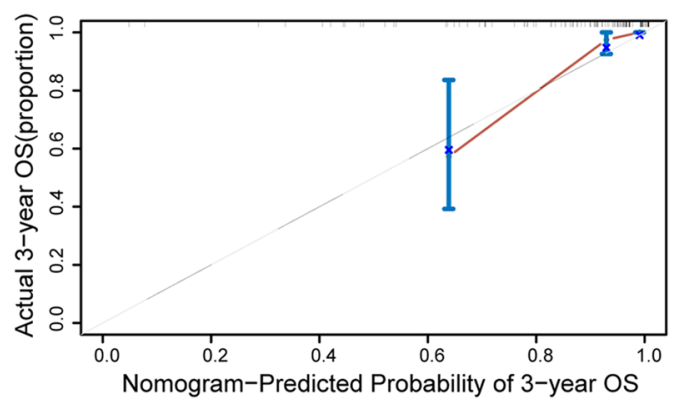

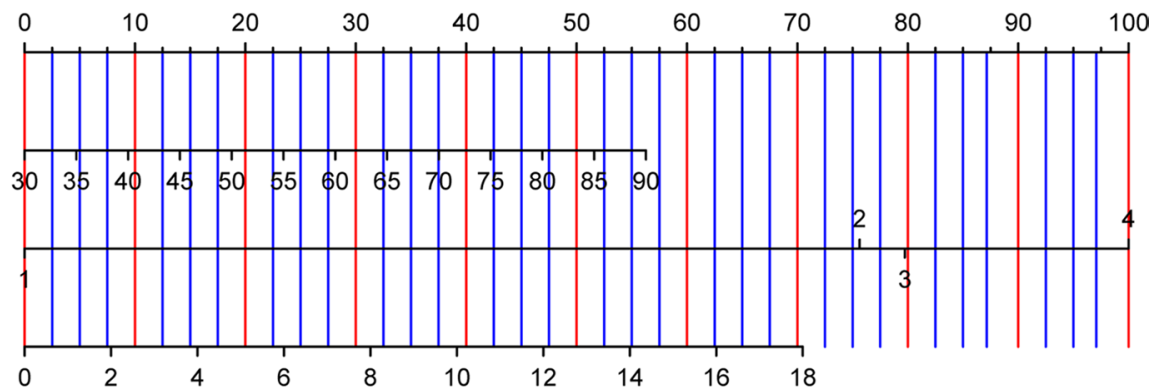
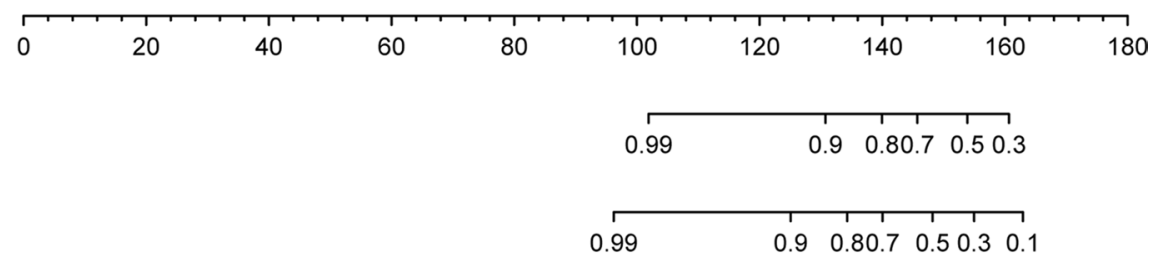

(C)
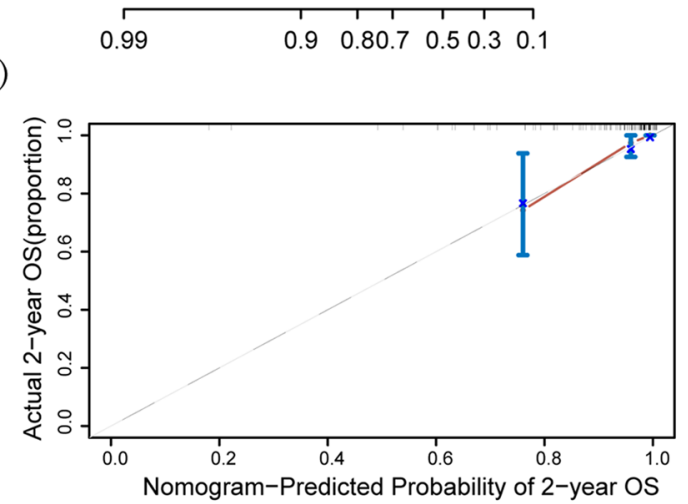

(E)

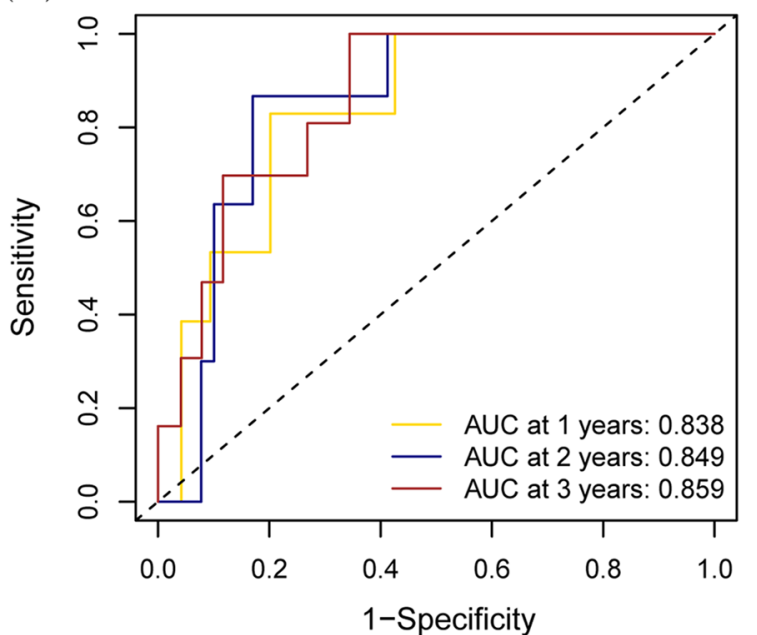

Fig. 8 Establishment and evaluation of a nomogram incorporating prognostic model and clinical features in READ patients. A Construction of a nomogram combining risk score with clinicopathological characteristics (age and TNM stage). B-D Calibration plots for the nomogram on 1-, 2-, and 3-year survival probability in patients with rectal cancer. E Time-dependent ROC curves for the nomogram on 1-, 2-, and 3-year survival probability in patients with rectal cancer 


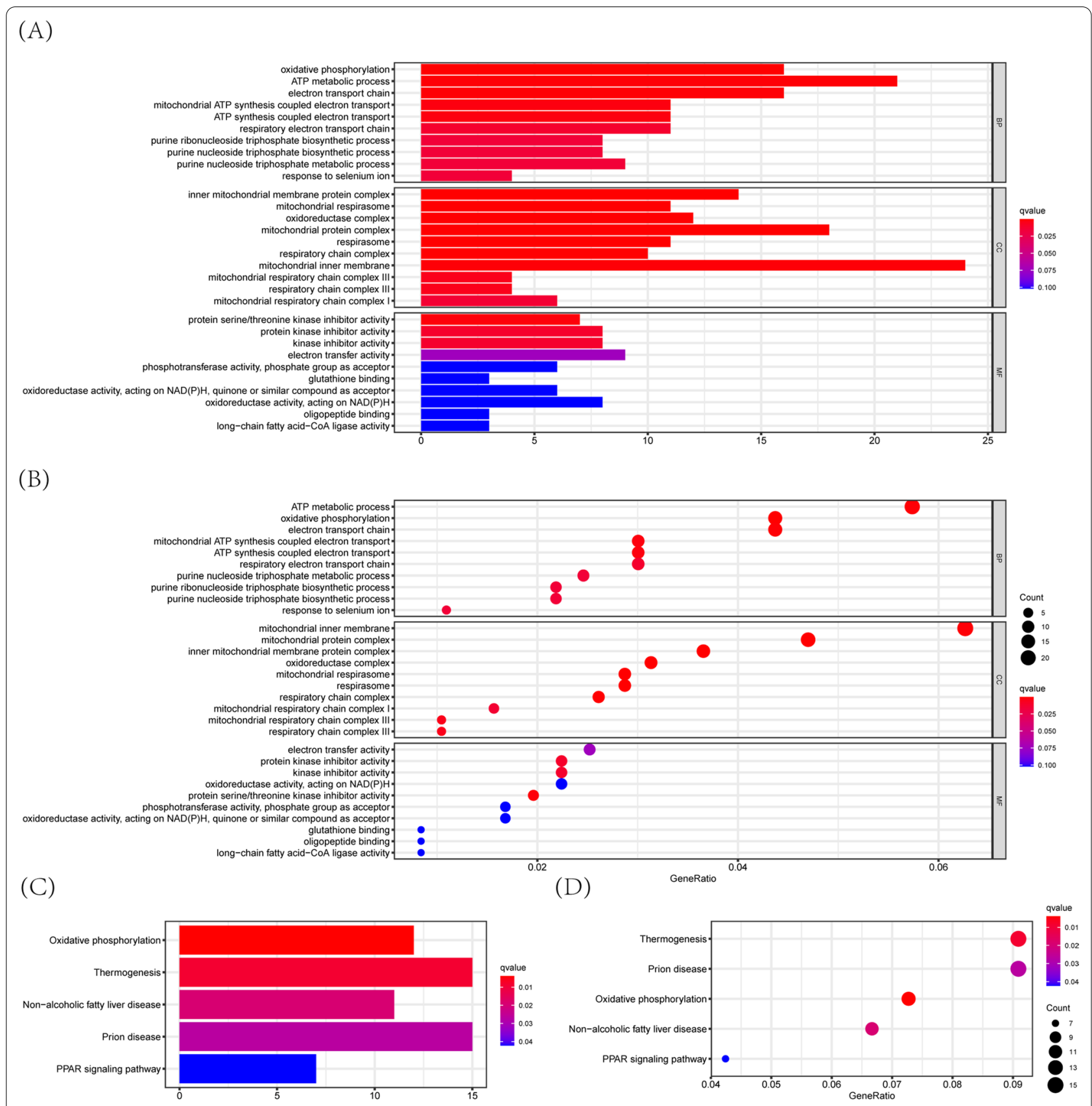

Fig. 9 Functional analyses between high-risk and low-risk groups in READ patients. A, B GO enrichment analysis on DEGs between high-risk and low-risk groups. C, D KEGG pathway analysis on DEGs between high-risk and low-risk groups

$p$ value $>0.05$ according to GSE17538 database (Additional file 1: Fig. S3B). Although the above results were inconsistent with the results of READ, we consider that the glycolysis-related prognostic model of READ might be appropriate for the high-throughput gene expression database not for microarray gene expression database in READ. In future, with new READ high-throughput gene expression database available for public, the glycolysis-related prognostic model of READ might be validated. Besides, the exact biological functions of the predictive genes, $P K P 2$ and TSAT3, remain unclear in rectal cancer and need to be elucidated in our subsequent studies. 


\section{Conclusion}

Our study compared the glycolysis-related gene signature between COAD and READ for the first time, found that there existed different glycolysis-related prognostic genes between COAD and READ, and showed that the glycolysis-related prognostic model of COAD was not appropriate for READ. Our study identified two novel glycolysis-related genes (PKP2 and TSTA3) associated with the prognosis of rectal cancer patients, and further established a risk model based on two novel glycolysis-related genes to effectively predict the prognosis of rectal cancer patients. Our study provides insight into the potential role of glycolysis in the development of rectal cancer and requires further investigation.

\section{Supplementary Information}

The online version contains supplementary material available at https://doi. org/10.1186/s40246-022-00377-0.

Additional file 1. Supplementary information.

\section{Acknowledgements}

We would like to thank Dr. Jiaojiao Zheng for comments on the manuscript.

\section{Authors' contributions}

ZL and ZL contributed to conceptualization, methodology and writing - original draft preparation; ZL provided software; ZL, BW and FL contributed to validation; $Z \mathrm{~L}, Z \mathrm{~L}$ and $Y L$ contributed to formal analysis; $X Z$ and $Y Y$ contributed to investigation; $Y Y$ and WW provided resources; $Z L$ and $S L$ contributed to data curation and visualization; $X Z, Y L, Y Y$ and WW contributed to writing-review and editing; BW, FL and WF contributed to supervision; WF administrated the project; ZL and WF acquired funding. All authors have read and agreed to the published version of the manuscript.

\section{Funding}

This study was supported by the National Natural Science Foundation of China [No. 81672361, No. 81972702 and No. 82002535].

\section{Availability of data and materials}

The data and materials are available under the permission of author.

\section{Declarations}

Ethics approval and consent to participate

Not applicable.

\section{Consent for publication}

All authors of this paper consent for publishing the manuscript and figures in the journal.

\section{Competing interests}

The authors declare that they have no competing interests.

\section{Author details}

'Department of General Surgery, Peking University Third Hospital, 49 Huayuan North Road, Haidian District, Beijing, People's Republic of China. ${ }^{2}$ Department of Medical Oncology and Radiation Sickness, Peking University Third Hospital, Beijing, People's Republic of China.

Received: 5 May 2021 Accepted: 3 December 2021

Published online: 02 February 2022

\section{References}

1. Fitzmaurice C, Dicker D, Pain A, Hamavid H, Moradi-Lakeh M, MacIntyre MF, et al. The global burden of cancer 2013. JAMA Oncol. 2015; 1(4):505-27.

2. Arnold M, Abnet CC, Neale RE, Vignat J, Giovannucci EL, McGlynn KA, et al. Global burden of 5 major types of gastrointestinal cancer. Gastroenterology. 2020;159(1):335-49.e15.

3. Dekker E, Tanis PJ, Vleugels JLA, Kasi PM, Wallace MB. Colorectal cancer. Lancet. 2019:394(10207):1467-80.

4. Deng Y. Rectal cancer in Asian vs. western countries: why the variation in incidence? Curr Treat Options Oncol. 2017;18(10):64.

5. Keum N, Giovannucci E. Global burden of colorectal cancer: emerging trends, risk factors and prevention strategies. Nat Rev Gastroenterol Hepatol. 2019;16(12):713-32.

6. Arnold M, Sierra MS, Laversanne M, Soerjomataram I, Jemal A, Bray F. Global patterns and trends in colorectal cancer incidence and mortality. Gut. 2017;66(4):683-91.

7. Collaborators GBDCC. The global, regional, and national burden of colorectal cancer and its attributable risk factors in 195 countries and territories, 1990-2017: a systematic analysis for the Global Burden of Disease Study 2017. Lancet Gastroenterol Hepatol. 2019;4(12):913-33.

8. Chen W, Zheng R, Baade PD, Zhang S, Zeng H, Bray F, et al. Cancer statistics in China, 2015. CA Cancer J Clin. 2016;66(2):115-32.

9. Cancer Genome Atlas N. Comprehensive molecular characterization of human colon and rectal cancer. Nature. 2012:487(7407):330-7.

10. De Sousa EMF, Wang $X$, Jansen M, Fessler E, Trinh A, de Rooij LP, et al. Poor-prognosis colon cancer is defined by a molecularly distinct subtype and develops from serrated precursor lesions. Nat Med. 2013;19(5):614-8.

11. Guinney J, Dienstmann R, Wang X, de Reyniès A, Schlicker A, Soneson C, et al. The consensus molecular subtypes of colorectal cancer. Nat Med. 2015:21(11):1350-6.

12. Sadanandam A, Lyssiotis CA, Homicsko K, Collisson EA, Gibb WJ, Wullschleger S, et al. A colorectal cancer classification system that associates cellular phenotype and responses to therapy. Nat Med. 2013;19(5):619-25.

13. Sun G, Li Y, Peng Y, Lu D, Zhang F, Cui X, et al. Identification of a five-gene signature with prognostic value in colorectal cancer. J Cell Physiol. 2019;234(4):3829-36.

14. Chen S, Cao G, Wu W, Lu Y, He X, Yang L, et al. Mining novel cell glycolysis related gene markers that can predict the survival of colon adenocarcinoma patients. Biosci Rep. 2020;40(8):BSR20201427.

15. Dienstmann R, Vermeulen L, Guinney J, Kopetz S, Tejpar S, Tabernero J. Consensus molecular subtypes and the evolution of precision medicine in colorectal cancer. Nat Rev Cancer. 2017;17(2):79-92.

16. Hanahan D, Weinberg RA. Hallmarks of cancer: the next generation. Cell. 2011;144(5):646-74.

17. Vander Heiden MG, Cantley LC, Thompson CB. Understanding the Warburg effect: the metabolic requirements of cell proliferation. Science. 2009:324(5930):1029-33.

18. Pavlova Natalya N, Thompson CB. The emerging hallmarks of cancer metabolism. Cell Metab. 2016;23(1):27-47.

19. Subramanian A, Tamayo P, Mootha VK, Mukherjee S, Ebert BL, Gillette MA, et al. Gene set enrichment analysis: a knowledge-based approach for interpreting genome-wide expression profiles. Proc Natl Acad Sci U S A. 2005;102(43):15545-50.

20. Wang G, Wang JJ, Yin PH, Xu K, Wang YZ, Shi F, et al. New strategies for targeting glucose metabolism-mediated acidosis for colorectal cancer therapy. J Cell Physiol. 2018;234(1):348-68.

21. Pate KT, Stringari C, Sprowl-Tanio S, Wang K, TeSlaa T, Hoverter NP, et al. Wnt signaling directs a metabolic program of glycolysis and angiogenesis in colon cancer. EMBO J. 2014;33(13):1454-73.

22. Harada K, Wu CC, Wang X, Mizrak Kaya D, Amlashi FG, Iwatsuki M, et al. Total lesion glycolysis assessment identifies a patient fraction with a high cure rate among esophageal adenocarcinoma patients treated with definitive chemoradiation. Ann Surg. 2020;272(2):311-8.

23. Liu C, LiY, Wei M, Zhao L, Yu Y, Li G. Identification of a novel glycolysisrelated gene signature that can predict the survival of patients with lung adenocarcinoma. Cell Cycle. 2019;18(5):568-79. 
24. Liu J, Li S, Feng G, Meng H, Nie S, Sun R, et al. Nine glycolysis-related gene signature predicting the survival of patients with endometrial adenocarcinoma. Cancer Cell Int. 2020;20(1):1-8.

25. Suh HY, Choi H, Paeng JC, Cheon GJ, Chung JK, Kang KW. Comprehensive gene expression analysis for exploring the association between glucose metabolism and differentiation of thyroid cancer. BMC Cancer. 2019;19(1):1260.

26. Tian G, Li G, Liu P, Wang Z, Li N. Glycolysis-based genes associated with the clinical outcome of pancreatic ductal adenocarcinoma identified by the cancer genome atlas data analysis. DNA Cell Biol. 2020;39(3):417-27.

27. Zhang C, Gou X, He W, Yang H, Yin H. A glycolysis-based 4-mRNA signature correlates with the prognosis and cell cycle process in patients with bladder cancer. Cancer Cell Int. 2020;20:177.

28. Rickelt S. Plakophilin-2: a cell-cell adhesion plaque molecule of selective and fundamental importance in cardiac functions and tumor cell growth. Cell Tissue Res. 2012;348(2):281-94.

29. Hao XL, Tian Z, Han F, Chen JP, Gao LY, Liu JY. Plakophilin-2 accelerates cell proliferation and migration through activating EGFR signaling in lung adenocarcinoma. Pathol Res Pract. 2019;215(7):152438.

30. Gao L, Li X, Guo Q, Nie X, Hao Y, Liu Q, et al. Identification of PKP 2/3 as potential biomarkers of ovarian cancer based on bioinformatics and experiments. Cancer Cell Int. 2020;20:509.

31. Zhang D, Qian Y, Liu X, Yu H, Zhao N, Wu Z. Up-regulation of plakophilin-2 is correlated with the progression of glioma. Neuropathology. 2017;37(3):207-16.

32. Takahashi H, Nakatsuji H, Takahashi M, Avirmed S, Fukawa T, Takemura M, et al. Up-regulation of plakophilin-2 and Down-regulation of plakophilin-3 are correlated with invasiveness in bladder cancer. Urology. 2012;79(1):240.e1-8

33. Arimoto K, Burkart C, Yan M, Ran D, Weng S, Zhang DE. Plakophilin-2 promotes tumor development by enhancing ligand-dependent and -independent epidermal growth factor receptor dimerization and activation. Mol Cell Biol. 2014;34(20):3843-54.

34. Niell N, Larriba MJ, Ferrer-Mayorga G, Sanchez-Perez I, Cantero R, Real FX, et al. The human PKP2/plakophilin-2 gene is induced by Wnt/betacatenin in normal and colon cancer-associated fibroblasts. Int J Cancer. 2018;142(4):792-804.

35. Tonetti M, Sturla L, Bisso A, Benatti U, De Flora A. Synthesis of GDP-Lfucose by the human FX protein. J Biol Chem. 1996;271(44):27274-9.

36. Zhou H, Sun L, Li J, Xu C, Yu F, Liu Y, et al. The crystal structure of human GDP-L-fucose synthase. Acta Biochim Biophys Sin (Shanghai). 2013;45(9):720-5

37. Miyoshi E, Moriwaki K, Nakagawa T. Biological function of fucosylation in cancer biology. J Biochem. 2008;143(6):725-9.

38. Miyoshi E, Moriwaki K, Terao N, Tan CC, Terao M, Nakagawa T, et al. Fucosylation is a promising target for cancer diagnosis and therapy. Biomolecules. 2012;2(1):34-45.

39. Shan M, Yang D, Dou H, Zhang L. Fucosylation in cancer biology and its clinical applications. Prog Mol Biol Transl Sci. 2019;162:93-119.

40. Wang M, Zhu J, Lubman DM, Gao C. Aberrant glycosylation and cancer biomarker discovery: a promising and thorny journey. Clin Chem Lab Med. 2019;57(4):407-16.

41. Zhang L, Gao Y, Zhang X, Guo M, Yang J, Cui H, et al. TSTA3 facilitates esophageal squamous cell carcinoma progression through regulating fucosylation of LAMP2 and ERBB2. Theranostics. 2020;10(24):11339-58.

42. Yang J, Kong P, Yang J, Jia Z, Hu X, Wang Z, et al. High TSTA3 expression as a candidate biomarker for poor prognosis of patients with ESCC. Technol Cancer Res Treat. 2018;17:1533033818781405.

43. Wang $Y$, Huang D, Chen KY, Cui M, Wang W, Huang X, et al. Fucosylation deficiency in mice leads to colitis and adenocarcinoma. Gastroenterology. 2017;152(1):193-205e10.

44. Zhao YP, Xu XY, Fang M, Wang H, You Q, Yi CH, et al. Decreased corefucosylation contributes to malignancy in gastric cancer. PLOS ONE. 2014;9(4):e94536.

45. Norton PA, Mehta AS. Expression of genes that control core fucosylation in hepatocellular carcinoma: systematic review. World J Gastroenterol. 2019;25(23):2947-60.

\section{Publisher's Note}

Springer Nature remains neutral with regard to jurisdictional claims in published maps and institutional affiliations.
Ready to submit your research? Choose BMC and benefit from:

- fast, convenient online submission

- thorough peer review by experienced researchers in your field

- rapid publication on acceptance

- support for research data, including large and complex data types

- gold Open Access which fosters wider collaboration and increased citations

- maximum visibility for your research: over $100 \mathrm{M}$ website views per year

At BMC, research is always in progress.

Learn more biomedcentral.com/submissions 